\title{
The Effect of Perceived Optimism On Body Image On Indian Young Adults
}

\author{
Sonia David \\ Jain University, India
}

\begin{abstract}
Purpose: The study aims to understand and determine how individuals' perceived optimism affects body image among young adults.

Methodology: The quantitative study uses an Ex Post Facto Correlational Design conducted on Indian young adults between 18-40 years old. The Body Image Avoidance Questionnaire (BIAQ) and the Positivity Scale was administered to the 508 participants through an online survey format. The quantitative analysis involved Pearson's Correlation Coefficient to measure the association between perceived optimism and body image.
\end{abstract}

Findings: The study findings imply sufficient evidence to conclude a linear relationship between perceived optimism and body image because the correlation coefficient is significantly different from 0 . The body image avoidance is weakly but negatively correlated with perceived optimism amongst the participants.

Study Implications: This study aids and contributes to the repertoire of body image and positive psychology research. It also helps explore individual differences in the trajectories of perceived optimism and body image as lockdown measures ease the understanding of the full psychological impact of this pandemic.

Keywords: perceived optimism; body image avoidance; young adults; lockdown; positive psychology. 